Trauma Berufskrankh 2013 · 15[Suppl 1]:52-58 DOI 10.1007/s10039-012-1878-5

Online publiziert: 28. Juni 2012

(c) Springer-Verlag Berlin Heidelberg 2012
K.-H. Frosch · A. Preiss

Chirurgisch-Traumatologisches Zentrum, Asklepios-Klinik St. Georg, Hamburg

\section{Knieverletzungen im Wachstumsalter} Vordere Kreuzbandruptur
Bis in die 1990er Jahre wurde die konservative Behandlung von Kreuzbandrupturen im Wachstumsalter favorisiert. Grund dafür war die Sorge um die noch nicht geschlossenen Wachstumsfugen, deren Verletzung eine Wachstumsstörung zur Folge haben kann.

Aktuell nimmt die Anzahl an Veröffentlichungen $\mathrm{zu}$, welche die operative Therapie der vorderen Kreuzbandruptur im Wachstumsalter bevorzugen, da mittels konservativer Behandlung oft unbefriedigende klinische Ergebnisse mit daraus resultierenden Spätschäden erzielt werden. So berichteten Barrack et al. [6] über 54\% schlechte Ergebnisse nach 38 Monaten. Instabilitäten nach konservativer Therapie wurden in bis zu $91 \%$ beobachtet [36]. In 50-75\% der Fälle kam es zu sekundären Meniskusläsionen nach Ruptur des vorderen Kreuzbandes (VKB; $[6,14,26])$. Bei persistierender Instabilität kann im Laufe der Zeit der Gelenkknorpel insbesondere posteromedial geschädigt werden [43]. Radiologisch nachweisbare degenerative Veränderungen fanden sich nach einer Follow-up-Zeit von 51 Monaten bei 11 von 18 Patienten [24]. Die Rate der Wiederaufnahme sportlicher Aktivität war nach konservativer Therapie eher gering (5-41\%; [38]).

Im Rahmen einer systematischen Literaturanalyse sollten folgende zentrale Fragen geklärt werden:

- Führt die operative Versorgung der vorderen Kreuzbandruptur im

Wachstumsalter zu guten klinischen

Ergebnissen?
- Können die oben angeführten Probleme der konservativen Therapie durch die operative Versorgung vermieden werden?

- Kommt es durch die operative Versorgung zu Sekundärschäden?

- Wo liegen die Grenzen der operativen Behandlung?

\section{Material und Methoden}

Es wurde eine selektive Literaturrecherche nach Veröffentlichungen von klinischen Beobachtungen über konservative und operative Behandlungen von Rupturen des vorderen Kreuzbandes bei Kindern und Jugendlichen im Zeitraum von Januar 1980 bis März 2009 in den Datenbanken PubMed ${ }^{\oplus}$, MEDLINE ${ }^{\oplus}$, The Cochrane Controlled Trial Register, EMBASE und MedPilot durchgeführt. Dabei wurde folgender Suchsyntax benutzt: ((„acl“ OR („anterior cruciate ligament “[mesh])) OR „reconstruction“) AND (((((„,child ${ }^{* “}$ OR „pediatric“ OR („open growth plates“ [mesh])) OR („growth age“[mesh])) OR („open physes“[mesh])) OR „young“) OR „immature“).
Außerdem wurden die Literaturnachweise der gefundenen Artikel nach weiteren relevanten Veröffentlichungen durchsucht, die bei der elektronischen Datensuche nicht gefunden wurden.

\section{Auswahlkriterien der Studien}

Berücksichtigung fanden alle Artikel, in denen Patienten mit offenen Wachstumsfugen und vorderer Kreuzbandruptur behandelt worden waren - sowohl konservativ als auch operativ. Als Einschlusskriterien galt das Vorliegen klinischer Ergebnisse, die anhand von Folgeuntersuchungen der Patienten evaluiert worden waren. Diese Ergebnisse mussten durch allgemeingültige Methoden z. B. den Lysholm-Score oder die IKDC-Kriterien (IKDC: „International Knee Documentation Committee") dargestellt worden sein [32]. Des Weiteren sollten Informationen über Komplikationen wie Beinlängendifferenzen oder Achsenabweichungen vorliegen.

Aus den Studien wurden Angaben über Alter und Anzahl der Patienten, Behandlungsart, Operationstechnik, Dauer der Folgeuntersuchungen, die klinischen
Tab. 1 Klinische Ergebnisse der konservativen vs. operativen Therapie ${ }^{a}$

\begin{tabular}{|lll}
\hline Klinisches Ergebnis & Konservative Therapie & Operative Therapie \\
\hline Lysholm-Score & $73,2 \pm 17,8$ & $95,5 \pm 3,1^{*}$ \\
\hline Giving-Way-Phänomen & $89,5 \pm 15,2 \%$ & $2,8 \pm 6,9 \%^{*}$ \\
\hline Rückkehr zum ursprünglichen Sportniveau & $30,9 \pm 33,8 \%$ & $91,2 \pm 3,1 \%{ }^{*}$ \\
\hline Positiver Lachman-Test Grad 2 oder 3 & $77,5 \pm 43,7 \%$ & $13,6 \pm 2,4 \%{ }^{*}$ \\
\hline${ }^{*} p<0,05^{a}$ Bei vorderer Kreuzbandruptur im Wachstumsalter & \\
\hline
\end{tabular}




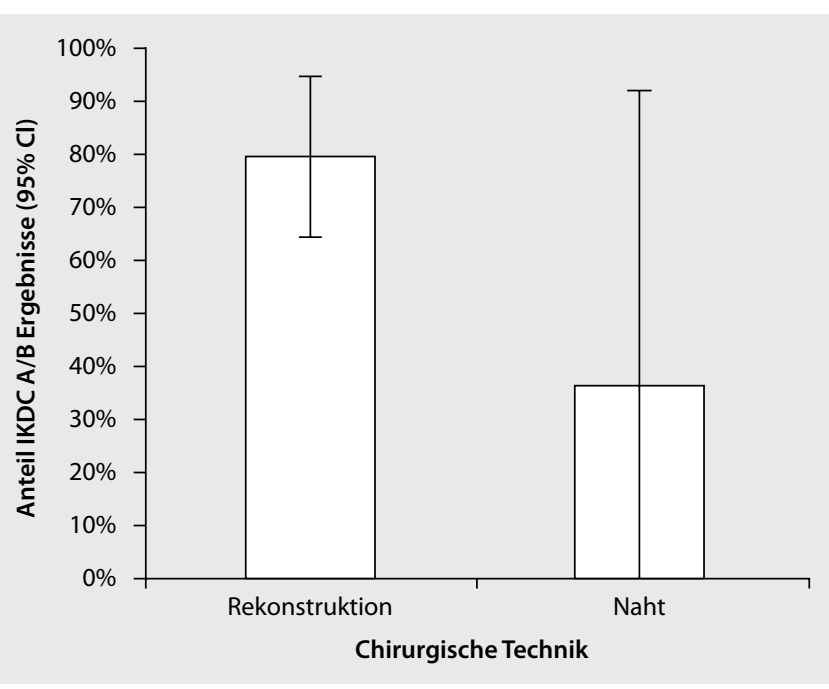

\section{Ergebnisse}

\section{Konservative Therapie}

Der Datensatz der Studien zu konservativ behandelten Patienten umfasste 10 Arbeiten (154 Patienten, 155 Knie; [1, 4, 10, 12, $14,20,21,27,29,44])$. Das mittlere Patientenalter lag bei 12,3 Jahren $(10,0$ bis 14,4 Jahre). Die Dauer bis zur Nachuntersuchung der Patienten ergab einen Median von 29 Monaten (24 bis 72 Monate).

Bei keinem der konservativ behandelten Patienten wurden bei der Nachuntersuchung ein Beinlängenunterschied oder eine Achsenabweichung gesehen. Der Mittelwert des Lysholm-Scores der konservativ versorgten Kinder lag bei 73,2 $\pm 17,8$ Punkten (Median 76,1 Punkte). Die Wahrscheinlichkeit für das Auftreten eines Giving-Way-Phänomens lag im Mittel bei 89,5\% ( $\pm 15,2 \%)$. Der Mittelwert der konservativ versorgten Kinder, die ihren ursprünglichen Sportlevel wiedererlangen konnten, belief sich auf $30,9 \%$ ( $\pm 33,8 \%$; Median $24,7 \%$ ) und derjenige der konservativ versorgten Kinder, die bei der Nachuntersuchung einen positiven Lachman-Test Grad 2 oder 3 aufwiesen, auf $77,5 \%( \pm 43,7 \%$; $\bullet$ Tab. 1$)$.

\section{Operative Therapie}

Der Datensatz der Studien zu operativ behandelten Patienten umfasste 55 Veröffentlichungen (935 Patienten, 941 Knie; [9]). Das mittlere Patientenalter betrug 13,4 Jahre (1,5 bis 16 Jahre). Das Intervall bis zur Nachuntersuchung der Patienten dauerte im Median 40 Monate (14 bis 89 Monate).

Der Mittelwert des Lysholm-Scores der operativ versorgten Kinder lag bei 95,5 $\pm 3,1$ Punkten (Median 96,5 Punkte). Die Wahrscheinlichkeit für das Auftreten eines Giving-Way-Phänomens betrug im Mittel 2,8\% ( $\pm 6,9 \%)$. Der Mittelwert der operativ versorgten Kinder, die ihren ursprünglichen Sportlevel wiedererlangen konnten, lag bei $91,2 \%( \pm 3,1 \%$; Median 96,5\%) und derjenige der operativ versorgten Kinder, die bei der Nachuntersuchung einen positiven LachmanTest Grad 2 oder 3 aufwiesen, bei 13,6\% $( \pm 2,4 \%$; 0 Tab. 1$)$. 


\section{Kreuzbandnaht vs. Rekonstruktion}

Die Kreuzbandnaht zeigte kein Fehlwachstum (0/69), der Lysholm-Score lag bei durchschnittlich 79,6 Punkten. Verglichen mit der Kreuzbandrekonstruktion zeigten sich bei 18/706 Kniegelenken eine Beinlängendifferenz oder eine Achsenabweichung [2,5\%, 95\%-CI (95\%-Konfidenzintervall): 1,5-4,0\%]. Die Rerupturrate betrug bei der Kreuzbandnaht 2/69 (2,9\%, 95\%-CI: 3,5-10,1\%), bei der Rekonstruktion 26/706 (3,7\%, 95\%-CI: 2,4$5,3 \%)$.

Das Outcome nach IKDC-Score ist in - Abb. 1 dargestellt.

\section{Fugen schonender vs. transphysärer Ersatz des vorderen Kreuzbands}

Zum Schutz der femoralen Fuge wurde entweder die Over-the-Top-Technik [16] oder Verfahren mit Fugen schonenden Bohrkanalanlagen verwendet [12]. Um die tibiale Fuge zu schonen, wurde das Transplantat entweder in Over-the-Front-Position unter dem Lig. transversum [16] oder via rein epiphysärem Tunnel eingebracht [2]. Andere Techniken wurden für den Fugen schonenden Ersatz nur selten verwendet.

Die Rate an postoperativ beobachteten Achsabweichungen oder Beinlängendifferenzen mit Fugen schonenden Techniken betrug 8/139 (5,8\%, 95\%-CI: 2,5-11,0\%) und mit transphysären Methoden 12/621 (1,9\%, 95\%-CI: 1,0-3,4\%; - Abb. 2).

Rerupturen traten in 2/139 Fällen (1,4\%, 95\%-CI: $0,2-5,1 \%)$ bei der transphysären und in $26 / 621$ Fällen $(4,2 \%$, 95\%-CI: 2,8-6,1\%) bei der Fugen schonenden Technik auf.

Ein Unterschied im klinischen Outcome zwischen den beiden Methoden fand sich nicht (• Abb. 3).

\section{Gelenknahe vs. gelenkferne Transplantatfixation}

Das Risiko des Auftretens von postoperativen Beinlängendifferenzen oder Achsenabweichungen bei gelenknaher vs. -ferner Transplantatfixation betrug $2 / 62$ (3,2\%, 95\%-CI: $0,4-11,2 \%)$ vs. $8 / 591(1,4 \%$, 95\%-CI: 0,6-2,7\%). Die Rerupturraten waren bei beiden Verfahren ähnlich hoch (3/62, $4,8 \%, 95 \%$-CI: $1,0-13,5 \%$ vs. $4,2 \%$, 95\%-CI: $2,8-6,2 \%)$.

Trauma Berufskrankh $2013 \cdot 15$ [Suppl 1]:52-58

c) Springer-Verlag Berlin Heidelberg 2012

\section{K.-H. Frosch · A. Preiss}

\section{Knieverletzungen im Wachstumsalter. Vordere Kreuzbandruptur}

\section{Zusammenfassung}

Zur Frage der operativen vs. konservativen Versorgung der vorderen Kreuzbandruptur im Wachstumsalter wurde eine systematische Literaturanalyse durchgeführt. Ausgewertet wurden 10 Studien mit insgesamt 154 Patienten zur konservativen und 55 Studien mit 935 Patienten zur operativen Therapie. Unter Ersterer war in $89,5 \pm 15,2 \%$ der Fälle ein Giving-Way-Phänomen zu beobachten, nur 30,9 $\pm 33,8 \%$ der Patienten kehrten zu ihrem ursprünglichen Sportniveau zurück. Nach operativer Versorgung fand sich in

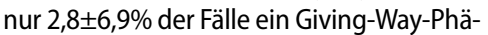
nomen, und $91,2 \pm 3,1 \%$ der Patienten erlangten wieder ihr ursprüngliches Sportniveau $(p<0,05)$. Das relative Risiko für eine Beinlängendifferenz $\geq 1 \mathrm{~cm}$ bzw. eine Achsabwei- chung $\geq 3^{\circ}$ nach operativer Behandlung war mit etwa $2,1 \%$ gering. Rerupturen traten in etwa 3,8\% der Fälle auf. Die Kreuzbandnaht induzierte kein Fehlwachstum, der LysholmScore war mit durchschnittlich 79,6 Punkten und oft persistierender Instabilität unbefriedigend. Implantate auf Fugenhöhe, Verwendung des Lig. patellae, implantatfreier Ersatz, Verletzung der ventralen tibialen Apophyse oder zu weit dorsal liegende femorale Kanäle gingen mit einer deutlich erhöhten Rate an Wachstumsstörungen einher.

\section{Schlüsselwörter}

Knie $\cdot$ Knieverletzungen Vorderes

Kreuzband . Wachstumsalter .

Wachstumsstörung

\section{Knee injuries in children and adolescents. Anterior cruciate ligament}

\section{Abstract}

The aim of the study was to examine whether a rupture of the anterior cruciate ligament $(A C L)$ in patients with open growth plates should be treated conservatively or operatively. An electronic search was performed in MEDLINE, the Cochrane controlled trial register, Embase, and Medpilot. Ten studies including 154 patients were analyzed regarding conservative treatment and 55 studies including 935 patients regarding operative treatment. The mean age for the conservative and operative treatment groups was 12.3 (10-14.4) years and $13.3(10-15.8)$ years, respectively. Overall, there were significantly better results concerning Lysholm score $(95.5$ vs. $73.2 ; p<0.05)$, instability described by the giving way phenomenon ( 2.8 vs. $89.5 \%$; $\mathrm{p}<0.05)$, and regaining former sports activity level $(91.2$ vs. $30.9 \%$; $p<0.05)$ in the operatively treated group. Relative risk for leg length differences more than $1 \mathrm{~cm}$ or axis deviation more than $3^{\circ}$ after surgery was low (2.1\%). Reruptures occurred in $3.8 \%$ of the cases. The analyzed data clearly show that operative treatment is superior to conservative management of $\mathrm{ACL}$ ruptures in children and adolescents.

\section{Keywords}

Knee $\cdot$ Knee injuries Anterior cruciate ligament · Adolescence · Growth disturbance

\section{Hamstring- vs. Knochen-Sehne- Knochen-Transplantat}

Das Risiko des Auftretens von postoperativen Beinlängendifferenzen oder Achsenabweichungen unter Verwendung eines BTB-Transplantats (BTB: ,bone tendon bone") vs. Hamstring-Sehnen betrug 6/166 (3,6\%, 95\%-CI: 1,3-7,7\%) vs. 9/458 (1,9\%, 95\%-CI: 0,9-3,7\%; D Abb. 4). Die Rerupturrate lag bei $4 / 166$ (2,4\%, 95\%CI: $0,7-6,1 \%)$ vs. $20 / 453$ (4,4\%, 95\%-CI: 2,7-6,7\%) für die Versorgung mit BTBvs. Hamstring-Transplantaten. Normale und fastnormale IKDC-Werte wurden in
46/54 mittels BTB- ( $85,2 \%, 95 \%$-CI: $72,9-$ 93,3\%) und in 54/67 mittels HamstringTransplantat versorgten Patienten beobachtet (80,6\%, 95\%-CI: 69,1-89,2\%).

\section{Diskussion}

Outcome nach operativer vs. konservativer Therapie

Die vorliegende Arbeit präsentiert Daten im Rahmen einer systematischen Literaturanalyse zur Therapie der vorderer Kreuzbandruptur im Wachstumsalter. 

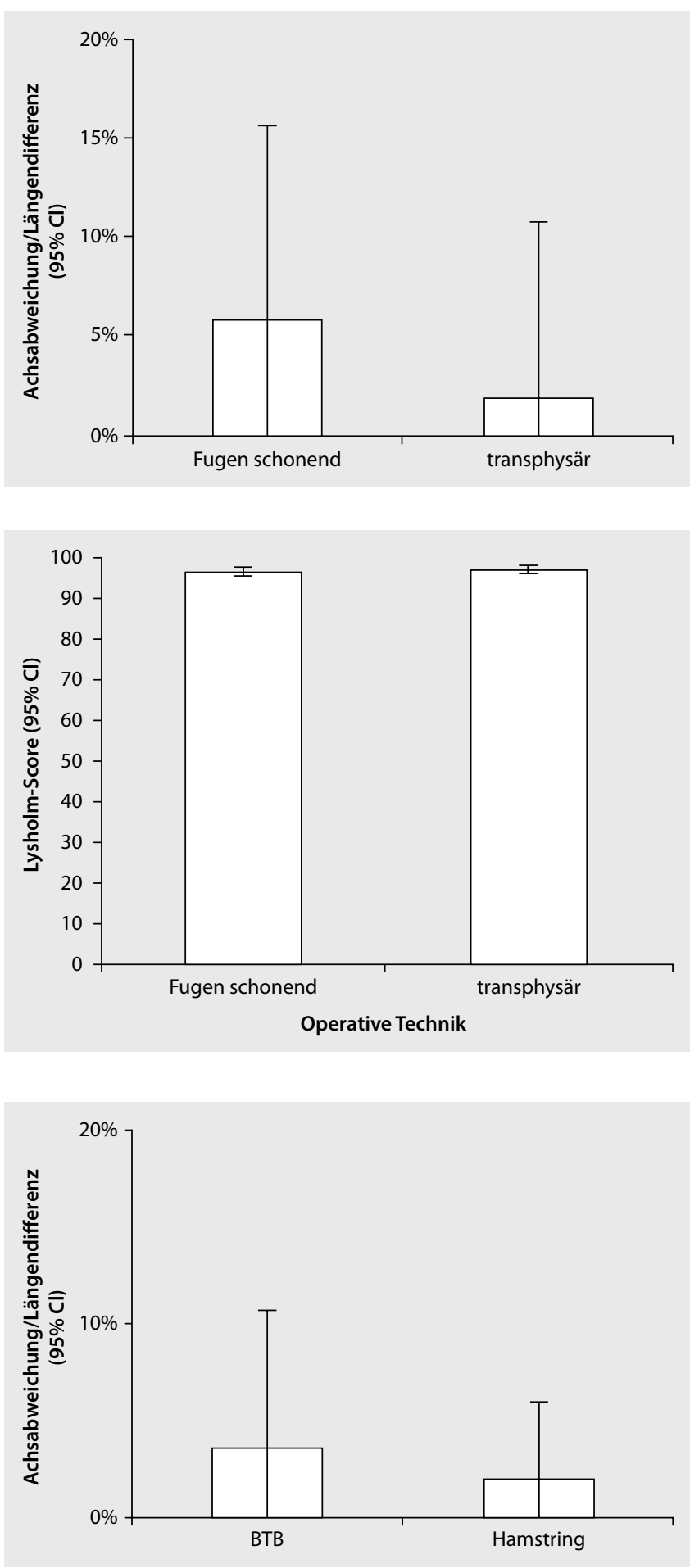

Abb. $2 \varangle$ Vergleich des Auftretens von postoperativen Beinlängendifferenzen oder Achsenabweichungen nach Fugen schonender vs. transphysärer Technik beim vorderen Kreuzbandersatz im Wachstumsalter, $95 \%$ Cl 95\%-Konfidenzintervall

Abb. $3 \varangle$ Vergleich der Lysholm-Scores zwischen Fugen schonender und transphysärer Technik beim vorderen Kreuzbandersatz im Wachstumsalter, 95\% Cl 95\%-Konfidenzintervall

Abb. $4 \varangle$ Vergleich des Auftretens von postoperativen Beinlängendifferenzen oder Achsenabweichungen nach Verwendung von BTB- vs. Hamstring-Transplantaten beim vorderen Kreuzbandersatz im Wachstumsalter, BTB "bone tendon bone", 95\% Cl 95\%-Konfidenzintervall

Die Analyse belegt, dass die operative der konservativen Therapie hinsichtlich des funktionellen Outcomes überlegen ist. So lag selbst der höchste nach konservativer Behandlung erreichte Lysholm-Score $(88,0)$ [29] unter dem Mittelwert $(95,5)$ nach operativer Rekonstruktion des vorderen Kreuzbandes. Bei den operativ versorgten Patienten zeigte sich auch länger-

fristig nach der Operation ein meist anhaltend gutes Ergebnis, was ebenfalls für die operative Therapie spricht.

Bezüglich des Giving-Way-Phänomens konnte nach operativer Versorgung meist ein gutes bis sehr gutes Ergebnis erzielt werden. Auch hier lag bei der Analyse der Daten das Maximum (16,7\%) der bei den operierten Patienten im Wachs- tumsalter auftretenden Giving-Way-Phänomene deutlich unter dem Mittelwert der konservativ therapierten Patienten (89,5\%). Kritisch anzumerken ist hier jedoch, dass evtl. nicht alle der teilweise sehr jungen Patienten adäquate Angaben zum subjektiven Giving-Way-Empfinden machen konnten.

Die Auswertung des Sportniveaus, welches sowohl vor als auch nach der Kreuzbandruptur evaluiert wurde, zeigt ebenfalls einen signifikanten Unterschied zugunsten der operativen Therapie. Wie bereits beim Lysholm-Score und beim Giving-Way-Phänomen lag der Anteil der Patienten, die ihr ursprüngliches Sportniveau wiedererlangen konnten, in der operierten Gruppe (91,2\%) über dem Maximalwert der konservativ versorgten Gruppe (85,7\%; [12]). Nur in 3 der 27 sich auf die operative Therapie beziehenden Studien blieben die Patienten im Mittel unter $80 \%$ ihres ursprünglichen Sportniveaus $[2,22,34]$. Nur ein Untersucher konnte bei den konservativ behandelten Patienten einen Wert von über $80 \%$ ermitteln [12]. Mit zunehmender Aktualität des Publikationsjahrs nahm die Zahl der Patienten nach konservativer Behandlung, die ihr ursprüngliches Sportniveau wieder erreichten, zu. Dies lässt sich vermutlich mit den sich im Laufe der Zeit verbessernden Behandlungsmethoden erklären.

Auch die Resultate des Lachman-Tests implizieren ein schlechteres Outcome nach konservativen Therapie.

Die gewonnenen Daten lassen deutlich erkennen, dass die operative Therapie bei der vorderen Kreuzbandruptur im Wachstumsalter zu präferieren ist. Jedoch können die chirurgischen Techniken, die bei Erwachsenen routinemäßig erfolgreich angewendet werden, nicht ohne weiteres auf Kinder und Jugendliche übertragen werden.

\section{Operative Therapie}

Die Frage nach der Verwendung von Fugen schonenden Techniken oder Verfahren, bei denen die Kanäle für das Transplantat direkt durch Wachstumsfugen gelegt werden (transphysär), ist anhand der vorliegenden Literaturanalyse eher zugunsten der transphysären Techniken zu beantworten. Fast die Hälfte der Fehl- 
wachstumsraten nach Fugen schonenden Techniken ist auf ein Genu recurvatum zurückzuführen. Für dessen Entstehung sind mehrere Ursachen zu diskutieren. Zum einen könnten durch die Anlage des tibialen Bohrkanals die Apophyse der proximalen Tibia oder der ventrale Abschnitt der proximalen Wachstumsfuge der Tibia verletzt werden und dadurch zu einem frühzeitigen Fugenschluss führen $[15,38]$. Grundsätzlich ist in diesem Zusammenhang auch daran zu denken, dass durch das fugennahe, tangentiale Bohren eine Hitzeschädigung der Epiphyse mit der Folge eines vorzeitigen Verschlusses möglich wäre [42]. Eine weitere Möglichkeit der Ausbildung eines Genu recurvatum, insbesondere bei den Fugen schonenden Techniken, wäre dadurch denkbar, dass durch das ventrale Herausziehen eines Transplantats aus dem Kniegelenk proximal und seiner Fixation distal der tibialen Fuge deren Wachstum ventralseitig durch das Transplantat blockiert werden könnte.

Die weitere Analyse der Entstehung eines Fehlwachstums nach VKB-Plastik im Wachstumsalter zeigt, dass eine beschriebene Beinverkürzung durch „stapels"verursacht wurde, mit denen jeweils die tibiale und die femorale Fuge fixiert wurden [18]. Auch wurde eine Beinverkürzung bei kongenitaler Anisomelie mit begleitender Kreuzbandinsuffizienz beobachtet [25] und eine weitere nach kontralateraler Femurfraktur [30]. Bei der Beurteilung der Beinlänge besteht jedoch bei den meisten Arbeiten das Problem, dass diese vor der Operation nicht exakt bestimmt wurde $[2,40]$, sodass die Beinlängendifferenz oft nicht mit letzter Sicherheit auf die Kreuzbandoperation zurückgeführt werden kann [2].

Wir wollten in vorliegender Arbeit insbesondere postoperative Achsenabweichungen oder Beinlängendifferenzen nach VKB-Pastik erfassen und analysieren. Wir legten deshalb eine kritische Grenze für ein Fehlwachstum fest, die bei $\geq 1 \mathrm{~cm}$ Beinlängendifferenz bzw. bei $\geq 3^{\circ}$ Achsenabweichung zur Gegenseite in der Frontal- und Sagittalebene lag. Von den insgesamt $29[2,3,17,18,22,25$, $30,38,40]$ von 860 Patienten, die nach unseren Kriterien ein Fehlwachstum aufwiesen, wurde dieses nur bei 13 Patienten vom jeweiligen Autor als relevant eingestuft, bei 16 Patienten, die nach den Kriterien der vorliegenden Studie ein Fehlwachstum aufwiesen, wurde dies von den entsprechenden Autoren zwar beobachtet und beschrieben, jedoch nicht als klinisch relevant bezeichnet [3].

Die beschriebenen Grenzen für ein Fehlwachstum wurden von uns so niedrig gewählt, um möglichst alle Patienten, für die in der Literatur ein Fehlwachstum beschrieben wurde, zu erfassen und die Dunkelziffer möglichst niedrig zu halten. Von einigen Autoren wurden die Grenzen für ein Fehlwachstum jedoch deutlich höher gesetzt als in vorliegender Studie [40], sodass nicht alle Wachstumsstörungen auf dem hier geforderten Level in die vorliegende Studie aufgenommen werden konnten. Bei einer Fehlwachstumsrate von insgesamt $2,1 \%$, wie sie in vorliegender Studie aufgezeigt wurde, ist deshalb von einer zusätzlichen Dunkelziffer auszugehen.

Von allen Autoren [2, 3, 17, 18, 22, 25, $30,38,40]$, die in ihren Studien Wachstumsstörungen beobachteten, gaben nur 2 einen Grund für die Entstehung der Wachstumsstörung an.

Das in der Literatur insgesamt 4-mal beschriebene Genu valgum kann durch das Implantatmaterial hervorgerufen werden, mit dem eine Wachstumsfuge lateral am Femur perforiert wurde [17]. Dreimal wurden zu seiner Entstehung keine Angaben gemacht. Aus Tierversuchen [39] ist bekannt, dass ein zu randständiges transphysäres Bohren des femoralen Kanals mit einer Verletzung des Ranvier-Schnürrings einhergehen kann, was zu einem frühzeitigen Fugenschluss am lateralen Femur und dadurch zu einem Genu valgum führen kann [39]. Es ist deshalb darauf zu achten, dass bei der femoralen Anlage des Bohrkanals nach dorsal noch eine ausreichend breite Knochenbrücke stehen bleibt. Auch ist es grundsätzlich wichtig, dass ein Weichteiltransplantat im Bohrkanal auf Höhe der Fugen zu liegen kommt, da durch leere Bohrkanäle ebenfalls ein Fehlwachstum mit frühzeitigem Fugenschluss hervorgerufen werden kann [39].

Bezüglich der Frage der gelenknahen oder -fernen Fixation muss im Rahmen dieser Studie eindeutig für eine gelenkferne Transplantatfixation plädiert werden. Die klinischen Ergebnisse zeigten zwischen beiden Gruppen keine wesentlichen Unterschiede, in Einzelfällen jedoch wurden durch ein fugennahes oder -kreuzendes Implantat Wachstumsstörungen induziert $[17,18]$.

Von einigen Autoren wird empfohlen, die Wachstumsfuge möglichst steil zu perforieren, um ein Fehlwachstum zu verhindern [42]. Dies würde bedeuten, den femoralen Bohrkanal transtibial anzulegen und nicht über das anteromediale Portal. In vorliegender Arbeit jedoch zeigten beide Verfahren ähnliche klinische Ergebnisse, die anteromediale Technik war nicht mit höheren Fehlwachstumsraten assoziiert $[2,3,11,19,33]$. Die Anlage des femoralen Bohrkanals über das anteromediale Portal halten wir deshalb auch bei offenen Wachstumsfugen für erlaubt.

$\mathrm{Ob}$ auch Beinlängendifferenzen durch eine optimierte Operationstechnik im Rahmen der vorderen Kreuzbandersatzplastik im Wachstumsalter vermieden werden können, ist derzeit noch unklar. Es ist zwar tierexperimentell beschrieben, dass durch ein geeignetes operatives Vorgehen die Entstehung von Fehlwachstum und insbesondere von Beinlängendifferenzen umgangen werden kann [23], hier ist jedoch der Beobachtungszeitraum von 24 Wochen als eher kurz anzusehen. Bei einem statistischen Risiko von etwa $2,0 \%$, posteroperativ eine Beinlängendifferenz von $\geq 1 \mathrm{~cm}$ zu erleiden, ist es grundsätzlich fraglich, ob dies im Tierversuch bei kleinen Tierzahlen überhaupt evident werden kann. Da histologisch im Bohrkanalbereich auch ohne nachfolgendes Fehlwachstum eine Reaktion der Epiphysenfuge nachweisbar ist [23], kann eine Stimulation der Wachstumsfuge mit nachfolgender Stimulation des Längenwachstums im Einzelfall wahrscheinlich nur schwer vermieden werden. In diesem Zusammenhang ist eine Studie von McIntosh et al. [22] interessant, die durch exakte Vermessung eine durchschnittliche Beinverlängerung des operierten Beines von $6,2 \mathrm{~mm}$ feststellten, wobei 15 von 16 untersuchten Patienten Unterschiede in der Beinlänge aufwiesen. 


\section{Postoperatives Outcome bei Kindern}

Die Gesamtschau der analysierten Literatur zeigt, dass mit der operativen Versorgung der intraligamentären vorderen Kreuzbandruptur in mehr als $80 \%$ der Fälle gute klinische Ergebnisse erzielt werden können. Im Vergleich zu Erwachsenen ist das Risiko, ein Fehlwachstum zu erleiden, zusätzlich zu berücksichtigen. Einzelne Autoren wiesen darauf hin, dass das klinische Ergebnis einer vorderen Kreuzbandplastik bei Kindern schlechter ausfällt als dasjenige von erwachsenen $\mathrm{Pa}$ tienten [38]. Bei entsprechender Operationstechnik können jedoch auch bei offenen Wachstumsfugen gute und sehr gute Ergebnisse erzielt werden, bei nur geringem Risiko eines Fehlwachstums [16, 32].

Die Interpretation der Ergebnisse und der hier vorliegenden Daten muss vor dem Hintergrund erfolgen, dass alle für diese Arbeit analysierten Studien einen Evidenzlevel von III oder IV aufwiesen. Prospektiv randomisierte Studien zum Thema vordere Kreuzbandruptur im Wachstumsalter beim Menschen existierten zum Zeitpunkt der Datenerhebung nicht.

\section{Fazit für die Praxis}

\section{Mit der vorderen Kreuzbandplastik im Wachstumsalter können gute klinische Ergebnisse erzielt werden. Das Risiko, eine Wachstumsstörung zu erleiden, be- trägt mindestens $2,1 \%$, insbesondere ist mit einer deutlich höheren Dunkelzif- fer an möglicherweise klinisch nicht oder wenig relevanten Beinverlängerungen zu rechnen. Eine exakte, auf das wach- sende Skelett abgestimmte Operations- technik sollte unbedingt beachtet wer- den.}

\section{Korrespondenzadresse}

\section{Prof. Dr. K.-H. Frosch}

Chirurgisch-Traumatologisches Zentrum, Asklepios-Klinik St. Georg, Lohmühlenstraße 5, 20099 Hamburg k.frosch@asklepios.com
Danksagung. Die Studie wurde in Zusammenarbeit mit dem Komitee Trauma der AGA erstellt. Die statistische Analyse wurde mit Unterstützung von PD Dr. Dirk Stengel, Unfallkrankenhaus Berlin Marzahn, und Peter Wohlmuth, Asklepios ProResearch St. Georg, Hamburg, durchgeführt.

Interessenkonflikt. Der korrespondierende Autor gibt für sich und seinen Koautor an, dass kein Interessenkonflikt besteht.

The supplement containing this article is not sponsored by industry.

\section{Literatur}

1. Aichroth PM, Patel DV, Zorrilla P (2002) The natural history and treatment of rupture of the anterior cruciate ligament in children and adolescents. A prospective review. J Bone Joint Surg Br 84:38-41

2. Anderson AF (2003) Transepiphyseal replacement of the anterior cruciate ligament in skeletally immature patients. A preliminary report. J Bone Joint Surg Am 85-A:1255-1263

3. Andrews M, Noyes FR, Barber-Westin SD (1994) Anterior cruciate ligament allograft reconstruction in the skeletally immature athlete. Am J Sports Med 22:48-54

4. Arbes S, Resinger C, Vecsei V et al (2007) The functional outcome of total tears of the anterior cruciate ligament $(A C L)$ in the skeletally immature patient. Int Orthop 31:471-475

5. Attmanspacher W, Dittrich V, Stedtfeld HW (2003) Results on treatment of anterior cruciate ligament rupture of immature and adolescents. Unfallchirurg 106:136-143

6. Barrack RL, Bruckner JD, Kneisl J et al (1990) The outcome of nonoperatively treated complete tears of the anterior cruciate ligament in active young adults. Clin Orthop Relat Res 259:192-199

7. Edwards PH, Grana WA (2001) Anterior cruciate ligament reconstruction in the immature athlete: long-term results of intra-articular reconstruction. Am J Knee Surg 14:232-237

8. Engebretsen L, Svenningsen S, Benum P (1988) Poor results of anterior cruciate ligament repair in adolescence. Acta Orthop Scand 59:684-686

9. Frosch KH, Stengel D, Brodhun T et al (2010) Outcomes and risks of operative treatment of rupture of the anterior cruciate ligament in children and adolescents. Arthroscopy 26:1539-1550

10. Graf BK, Lange RH, Fujisaki CK et al (1992) Anterior cruciate ligament tears in skeletally immature patients: meniscal pathology at presentation and after attempted conservative treatment. Arthroscopy $8: 229-233$

11. Hoffmann $F$ (1998) Versorgung der vorderen Kreuzbandruptur bei offenen Wachstumsfugen. Arthroskopie 11:28-33

12. Janarv PM, Nystrom A, Werner S et al (1996) Anterior cruciate ligament injuries in skeletally immature patients. J Pediatr Orthop 16:673-677

13. Kaeding CC, Flanigan D, Donaldson C (2010) Surgical techniques and outcomes after anterior cruciate ligament reconstruction in preadolescent patients. Arthroscopy 26:1530-1538

14. Kannus $P$, Jarvinen $M$ (1988) Knee ligament injuries in adolescents. Eight year follow-up of conservative management. J Bone Joint Surg Br 70:772776
15. Kocher MS et al (2002) Management and complications of anterior cruciate ligament injuries in skeletally immature patients: survey of the Herodicus Society and the ACL Study Group. J Pediatr Orthop 22(4):452-457

16. Kocher MS, Garg S, Micheli LJ (2005) Physeal sparing reconstruction of the anterior cruciate ligament in skeletally immature prepubescent children and adolescents. J Bone Joint Surg Am 87(11):2371-2379

17. Koman JD, Sanders JO (1999) Valgus deformity after reconstruction of the anterior cruciate ligament in a skeletally immature patient. A case report. J Bone Joint Surg Am 81(5):711-715

18. Lipscomb AB, Anderson AF (1986) Tears of the anterior cruciate ligament in adolescents. J Bone Joint Surg Am 68:19-28

19. Lukas C, Eberhardt O, Wirth T, Fernandez F (2007) Results of $A C L$ reconstruction with a periost-patella tendon-periost graft in growth age. $Z$ Orthop Unfall 145(6):706-711

20. Mccarroll JR, Rettig AC, Shelbourne KD (1988) Anterior cruciate ligament injuries in the young athlete with open physes. Am J Sports Med 16:44-47

21. Mccarroll JR, Shelbourne KD, Porter DA et al (1994) Patellar tendon graft reconstruction for midsubstance anterior cruciate ligament rupture in junior high school athletes. An algorithm for management. Am J Sports Med 22:478-484

22. Mcintosh AL, Dahm DL, Stuart MJ (2006) Anterior cruciate ligament reconstruction in the skeletally immature patient. Arthroscopy 22:1325-1330

23. Meller R et al (2008) Hindlimb growth after a transphyseal reconstruction of the anterior cruciate ligament: a study in skeletally immature sheep with wide-open physes. Am J Sports Med 36(12):24372443

24. Micheli LJ, Metzl JD, Di Canzio J et al (1999) Anterior cruciate ligament reconstructive surgery in adolescent soccer and basketball players. Clin J Sports Med 9:138-141

25. Micheli LJ, Rask B, Gerberg L (1999) Anterior cruciate ligament reconstruction in patients who are prepubescent. Clin Orthop Relat Res 364:40-47

26. Millett PJ, Willis AA, Warren RF (2002) Associated injuries in pediatric and adolescent anterior cruciate ligament tears: does a delay in treatment increase the risk of meniscal tear? Arthroscopy 18:955959

27. Mizuta H, Kubota K, Shiraishi M et al (1995) The conservative treatment of complete tears of the anterior cruciate ligament in skeletally immature patients. J Bone Joint Surg Br 77:890-894

28. Moher D, Cook DJ, Eastwood S et al (1999) Improving the quality of reports of meta-analyses of randomised controlled trials: the QUOROM statement. Quality of reporting of meta-analyses. Lancet 354:1896-1900

29. Moksnes H, Engebretsen L, Risberg MA (2008) Performance-based functional outcome for children 12 years or younger following anterior cruciate ligament injury: a two to nine-year follow-up study. Knee Surg Sports Traumatol Arthrosc 16:214-223

30. Nakhostine M, Bollen SR, Cross MJ (1995) Reconstruction of mid-substance anterior cruciate rupture in adolescents with open physes. J Pediatr Orthop 15(3):286-287

31. Pressman AE, Letts RM, Jarvis JG (1997) Anterior cruciate ligament tears in children: an analysis of operative versus nonoperative treatment. J Pediatr Orthop 17:505-511 


\section{Kindliches Trauma}

32. Risberg MA, Holm I, Steen H et al (1999) Sensitivity to changes over time for the IKDC form, the Lysholm score, and the Cincinnati knee score. A prospective study of $120 \mathrm{ACL}$ reconstructed patients with a 2-year follow-up. Knee Surg Sports Traumatol Arthrosc 7:152-159

33. Robert H, Bonnard C (1999) The possibilities of using the patellar tendon in the treatment of anterior cruciate ligament tears in children. Arthroscopy 15(1):73-76

34. Schneider FJ, Kraus T, Linhart WE (2008) Anterior cruciate ligament reconstruction with semitendinosus tendon in children. Oper Orthop Traumatol 20:409-422

35. Schultz A (1996) Ergebnisse der operierten vorderen Kreuzbandruptur bei Kindern. Acta Chir Austr 28:25-26

36. Seil R, Kohn D (2000) Ruptures of the anterior cruciate ligament $(\mathrm{ACL})$ during growth. Bull Soc Sci Med Grand Duche Luxemb 1:39-53

37. Seil R, Robert H (2004) Complete anterior cruciate ligament tears in children. Rev Chir Orthop Reparatrice Appar Mot 90:3S11-20

38. Seil R, Robert H (2005) VKB-Plastik bei offenen Wachstumsfugen. Arthroskopie 18:48-52

39. Seil R, Pape D, Kohn D (2008) The risk of growth changes during transphyseal drilling in sheep with open physes. Arthroscopy 24:824-833

40. Seon JK, Song EK, Yoon TR et al (2005) Transphyseal reconstruction of the anterior cruciate ligament using hamstring autograft in skeletally immature adolescents. J Korean Med Sci 20:1034-1038

41. Shelbourne KD, Gray T, Wiley BV (2004) Results of transphyseal anterior cruciate ligament reconstruction using patellar tendon autograft in Tanner stage 3 or 4 adolescents with clearly open growth plates. Am J Sports Med 32(5):1218-1222

42. Sobau C, Ellermann A (2004) Anterior cruciate ligament reconstruction with hamstring tendons in the young. Unfallchirurg 107(8):676-679

43. Strobel MW (2005) Vordere Kreuzbandinsuffizienz. In: Wirth CJ, Zichner L, Kohn D (Hrsg) Orthopädie und orthopädische Chirurgie. Thieme, Stuttgart New York, S 263-288

44. Woods GW, O'Connor DP (2004) Delayed anterior cruciate ligament reconstruction in adolescents with open physes. Am J Sports Med 32:201-210 